\title{
Enfermedad periodontal: ¿es factor de riesgo para parto pretérmino, bajo peso al nacer o preeclampsia?
}

\author{
José Luis Castaldi, ${ }^{1}$ Marta Susana Bertin, ${ }^{1}$ Fabián Giménez ${ }^{1}$ y Roberto Lede ${ }^{2}$
}

Forma de citar Castaldi JL, Bertin MS, Giménez F, Lede R. Enfermedad periodontal: ¿es factor de riesgo para parto pretérmino, bajo peso al nacer o preeclampsia? Rev Panam Salud Publica. 2006;19(4):253-8.

RESUMEN Objetivos. Evaluar si la enfermedad periodontal (EP) constituye un factor de riesgo de parto pretérmino, bajo peso al nacer o preeclampsia y si hay alguna relación entre las formas clínicas de la EP y los resultados obstétricos mencionados.

Métodos. Estudio analítico, transversal y prospectivo de todas las mujeres que tuvieron su parto en el Hospital Dr. José Penna, de la ciudad de Bahía Blanca, Argentina, entre el 1. ${ }^{\circ}$ de febrero y el 18 de julio de 2003 y entre el 1..$^{\circ}$ de marzo y el 31 de mayo de 2004. A las mujeres que cumplieron los criterios de inclusión (ser mayor de 18 años, tener al menos 18 dientes, no ser diabética y que los hijos hubieran sobrevivido al parto) se les practicó un examen odontológico en busca de hemorragia al sondaje, movilidad dentaria o inflamación gingival y se realizaron mediciones clínicas de la pérdida de inserción. Se calcularon las razones de posibilidades (odds ratios, RP) con sus intervalos de confianza del 95\% (IC95\%) y se ajustaron los resultados por las variables "ser fumadora" y "tener anemia".

Resultados. Se registraron 2003 nacimientos, correspondientes a 1982 partos. Se excluyeron $420(21,2 \%)$ mujeres por no cumplir los criterios de inclusión o por no poder realizárseles el diagnóstico odontológico previsto. De las 1562 mujeres evaluadas, 809 (51,8\%) presentaban alguna de las afecciones buscadas; de ellas, $274(17,5 \%)$ padecían EP grave y $535(34,3 \%)$ tenían gingivitis. En total, 149 (9,5\%) partos fueron pretérmino, en $161(10,3 \%)$ nacieron niños con bajo peso y en $157(10,0 \%)$ se presentaron casos de preeclampsia. No se observó asociación alguna entre la EP y el parto pretérmino $(R P=1,06 ;$ IC95\%: 0,74 a 1,50), el bajo peso al nacer $(R P=1,05 ;$ IC95\%: 0,74 a 1,47) y la preeclampsia $(R P=0,99 ;$ IC95\%: 0,70 a 1,40). El riesgo de dar a luz un niño con bajo peso al nacer en el subgrupo de fumadoras de más de 10 cigarrillos diarios fue mayor en las que tenían enfermedad periodontal grave que en las mujeres cuya salud bucal era buena ( $R P=3,81 ;$ IC95\%: 1,46 a 10,05; $\mathrm{P}=0,001)$. Se confirmó que la anemia es un factor de riesgo de bajo peso al nacer ( $R P=1,74 ;$ IC95\%: 1,03 a 2,94; $\mathrm{P}=0,02)$.

Conclusiones. No se encontró ninguna asociación significativa entre la EP y el parto pretérmino, el bajo peso al nacer o la preeclampsia.

Palabras clave Enfermedades periodontales, prematuro, recién nacido de bajo peso, preeclampsia, Argentina.

La enfermedad periodontal (EP) es una infección crónica producida por bacte-

\footnotetext{
Hospital Interzonal de Agudos Dr. José Penna, Bahía Blanca, Argentina. La correspondencia se debe dirigir a Marta Susana Bertin, Servicio de Tocoginecología, HIGA Dr. José Penna, Quillen 258,
}

rias anaerobias gramnegativas que crecen dentro del surco gingival. Diversos

8000 Bahía Blanca, Argentina. Correo electrónico: bertincastaldi@patagoniant.com.ar

2 Instituto Argentino de Medicina Basada en las Evidencias (IAMBE), Buenos Aires, Argentina. factores - genéticos, ambientales y biológicos, entre otros- favorecen la evolución a un proceso destructivo (1). Entre los factores biológicos, las hormonas sexuales femeninas desempeñan un papel muy importante en ese 
proceso, ya que su concentración aumenta considerablemente en el organismo durante el embarazo o por el uso de medicamentos anticonceptivos $(2,3)$. Este incremento provoca cambios en el organismo a nivel vascular, celular, microbiológico e inmunitario. Aunque la secreción de estas hormonas es cíclica, al final del tercer trimestre del embarazo alcanza concentraciones plasmáticas más elevadas, lo que afecta notablemente a las estructuras gingivo-periodontales.

En el tejido gingival existen receptores del estrógeno y de la progesterona. Los estrógenos se metabolizan en ese tejido y pasan de estrona a estradiol. La progesterona produce alteraciones clínicas en los capilares gingivales y puede provocar la llamada gingivitis gestacional, caracterizada por la presencia de eritemas, edemas, exudado gingival y el aumento de la permeabilidad capilar. La carga de bacterias aerobias y anaerobias en la cavidad bucal aumenta durante el embarazo $(4,5)$, especialmente de Prevolella intermedia, que utiliza la progesterona y el estradiol debido a su semejanza estructural con la vitamina $\mathrm{K}$ necesaria para su crecimiento (6).

Las concentraciones habituales de progesterona y estradiol durante el embarazo estimulan la síntesis de prostaglandinas en la encía de la embarazada (7-11). A esto se debe añadir que durante la infección periodontal aumenta aun más el número de microorganismos anaerobios gramnegativos que se acumula en las encías, así como las concentraciones de lipopolisacáridos y endotoxinas producidos por esos microorganismos. Además de las prostaglandinas E2, también se acumulan algunos mediadores de la inflamación, como los factores de necrosis tumoral $\alpha$ y la interleucina $1 \beta$ (12). La combinación de estos dos procesos puede llegar a constituir un riesgo para la integridad del feto y la placenta en mujeres con una respuesta inmunitaria celular reducida producto del embarazo (13-15).

En 1996, Steven Offenbacher propuso la teoría de que las bacterias que participan en la EP estimulan la síntesis y la liberación de prostaglandinas, por lo que podrían constituir un factor de riesgo de parto prematuro, bajo peso al nacer y preeclampsia (16). Sin embargo, otros autores sostienen que el aumento hormonal no agrava la EP durante el embarazo (17).

En los últimos años, muchos autores han investigado la posible asociación entre la EP y diversas enfermedades sistémicas, como las enfermedades cardiovasculares, la arteriosclerosis, los accidentes cerebrovasculares, las endocarditis bacterianas, las enfermedades respiratorias y las complicaciones del parto (18-23). En general, se ha observado una tendencia a la asociación, aunque no siempre de forma significativa.

Debido a que el tema es aún motivo de controversia, se realizó el presente trabajo con el objetivo de evaluar si la EP constituye un factor de riesgo de parto pretérmino, bajo peso al nacer o preeclampsia y si existe alguna relación entre las formas clínicas de la EP y los resultados obstétricos mencionados.

\section{MATERIALES Y MÉTODOS}

Se realizó un estudio analítico, transversal y prospectivo de todas las mujeres que tuvieron su parto en el Hospital Dr. José Penna, de la ciudad de Bahía Blanca, Argentina, entre el $1 .^{\circ}$ de febrero y el 18 de julio de 2003 y entre el 1. ${ }^{\circ}$ de marzo y el 31 de mayo de 2004. La población urbana y semirrural que acude a esta institución se caracteriza por tener un bajo nivel socioeconómico.

A las mujeres que aceptaron participar en el estudio se les realizó en las 72 horas posteriores al parto un examen odontológico completo por ocho odontólogos previamente entrenados y que trabajaron por parejas. En el examen clínico periodontal se buscó específicamente si la paciente presentaba hemorragia al sondaje, movilidad dentaria o inflamación gingival y se realizaron mediciones clínicas de la pérdida de inserción. La profundidad de la bolsa y la pérdida de inserción periodontal se midieron en seis sitios en cada pieza dental. Las discrepancias durante el diagnóstico se resolvieron con la participación de un tercer profesional. Para este estudio se utilizó una sonda periodontal Zeffiro ${ }^{\mathrm{MR}}$ No. 42 (LASCOD Spa, Italia).

El centro de cómputos del servicio de maternidad entregaba diariamente a los grupos de odontólogos la relación de mujeres que habían dado a luz, sin mencionar las características del parto y del recién nacido. Los criterios de inclusión fueron tener 18 años o más, haber dado a luz un niño vivo y tener más de 18 dientes. Se excluyó del estudio a las mujeres diabéticas.

\section{Variables y definiciones}

Preeclampsia: aparición durante el embarazo de proteinuria (> $30 \mathrm{mg} / \mathrm{dL}$ ) e hipertensión arterial $(\geq 140 / 90 \mathrm{~mm}$ $\mathrm{Hg}$ ).

Peso bajo al nacer: recién nacido con peso $<2500 \mathrm{~g}$.

Edad gestacional al nacimiento: tiempo de gestación determinado mediante examen físico, según el método de Capurro y col. (24).

Anemia: hemoglobina $<11 \mathrm{~g} / \mathrm{L}$ en el último trimestre del embarazo (si faltaba el dato, se tomó la concentración de hemoglobina medida en el puerperio inmediato).

Fumadora: si había consumido durante el último mes al menos un cigarrillo diario; entre las fumadoras se identificaron la que fumaban $\geq 10$ cigarrillos diarios.

Estado de salud bucal: a) EP grave: 4 dientes o más con al menos un sitio con pérdida de inserción de $3 \mathrm{~mm}$ o más; b) EP o gingivitis, cuando no se cumplieron los criterios de EP grave pero se observaban hemorragia e inflamación gingival en más de $25 \%$ de los sitios; c) saludable: cuando no se alcanzaron los criterios anteriores.

Se elaboró una base de datos digital mediante el programa Epi Info v. 6.3. Se calcularon las razones de posibilidades (odds ratio, RP) y sus intervalos de confianza de $95 \%$ (IC95\%). Los resultados se ajustaron en función de las variables (fumadora y anemia) mediante el método de análisis estratificado de Mantel-Haenszel. Esta investigación contó con la aprobación del Comité de Ética del Hospital Dr. José Penna. 


\section{RESULTADOS}

En el período estudiado se registraron 2003 nacimientos, correspondientes a 1982 partos (hubo 17 embarazos gemelares y 2 embarazos triples). Del total de mujeres que dieron a luz se excluyeron 420 (21,2\%); de ellas, 148 $(7,4 \%)$ eran menores de 18 años, 47 $(2,4 \%)$ tenían menos de 18 dientes, 27 $(1,4 \%)$ eran diabéticas (16 de ellas desde antes del embarazo y 11 gestacionales), 19 (1,0\%) tuvieron hijos que no sobrevivieron al parto y a 179 $(9,0 \%)$ no se les pudo realizar el diagnóstico odontológico.

La imposibilidad de realizar la evaluación odontológica se debió a que no se encontró a la paciente cuando el odontólogo fue a examinarla, ya sea porque había regresado al centro original de donde se transfirió o porque había abandonado el hospital antes de tiempo.

El examen odontológico reveló que 809 mujeres $(51,8 \%)$ de las 1562 evaluadas presentaban alguna de las afecciones buscadas; de ellas, $274(17,5 \%)$ padecían EP grave y 535 (34,3\%), gingivitis. El desenlace de los partos fue como sigue: 149 partos $(9,5 \%)$ fueron pretérmino, en $161(10,3 \%)$ nacieron niños con bajo peso y en $157(10,0 \%)$ la paciente sufrió preeclampsia. En algunos casos se observaron varios de estos problemas.

Cuando se analizaron los resultados se detectó un mayor número de partos pretérmino en las pacientes con EP grave $(12,4 \%)$ que en las que tenían gingivitis $(8,4 \%)$ y en las mujeres cuya salud bucal era buena (9,3\%). Los casos de bajo peso al nacer también fueron más frecuentes en hijos de mujeres con EP grave $(14,2 \%)$ que en mujeres con gingivitis $(8,6 \%)$ y en las clasificadas como saludables $(10,1 \%)$. Con relación a la preeclampsia, los resultados fueron similares: $10,2 \%$ en las mujeres con EP grave, 9,9\% en las que tenían gingivitis y $10,1 \%$ en las que tenían buena salud bucal (cuadros 1 y 2 ). Sin embargo, estas relaciones no fueron estadísticamente significativas.

Cuando se ajustó según el hábito de fumar, no hubo cambios significativos en la asociación de la EP con el

CUADRO 1. Desenlaces del parto según la salud bucal

\begin{tabular}{|c|c|c|c|c|c|c|c|}
\hline & \multirow{2}{*}{$\begin{array}{c}\text { No. } \\
\text { de casos }\end{array}$} & \multicolumn{2}{|c|}{ Parto pretérmino } & \multicolumn{2}{|c|}{ Peso bajo al nacer } & \multicolumn{2}{|c|}{ Preeclampsia } \\
\hline & & No. & $\%$ & No. & $\%$ & No. & $\%$ \\
\hline \multicolumn{8}{|l|}{ Enfermedad } \\
\hline periodontal & 809 & 79 & $(9,7)$ & 85 & $(10,5)$ & 81 & $(10,0)$ \\
\hline Gingivitis & 535 & 45 & $(8,4)$ & 46 & $(8,6)$ & 53 & $(9,9)$ \\
\hline Grave & 274 & 34 & $(12,4)$ & 39 & $(14,2)$ & 28 & $(10,2)$ \\
\hline Boca saludable & 753 & 70 & $(9,3)$ & 76 & $(10,1)$ & 76 & $(10,1)$ \\
\hline Total & 1562 & 149 & $(9,5)$ & 161 & $(10,3)$ & 157 & $(10,1)$ \\
\hline
\end{tabular}

CUADRO 2. Asociación entre la enfermedad periodontal y el desenlace de los partos ${ }^{\mathrm{a}}$

\begin{tabular}{|c|c|c|c|c|c|c|}
\hline & \multicolumn{2}{|c|}{ Pretérmino } & \multicolumn{2}{|c|}{ Peso bajo al nacer } & \multicolumn{2}{|c|}{ Preeclampsia } \\
\hline & $\mathrm{RP}$ & $1 \mathrm{C} 95 \%{ }^{\mathrm{b}}$ & $\mathrm{RP}$ & IC95\% & $\mathrm{RP}$ & IC95\% \\
\hline $\begin{array}{l}\text { Enfermedad } \\
\text { periodontal }\end{array}$ & 1,06 & $(0,74-1,50)$ & 1,05 & $(0,74-1,47)$ & 0,99 & $(0,70-1,40)$ \\
\hline Gingivitis & 0,90 & $(0,59-1,35)$ & 0,84 & $(0,56-1,25)$ & 0,98 & $(0,67-1,44)$ \\
\hline Grave & 1,38 & $(0,87-2,18)$ & 1,48 & $(0,96-2,28)$ & 1,01 & $(0,63-1,64)$ \\
\hline
\end{tabular}

a Razón de posibilidades.

b Intervalo de confianza de $95 \%$.

CUADRO 3. Asociación entre la enfermedad periodontal y el desenlace del parto, controlado por el hábito de fumar

\begin{tabular}{|c|c|c|c|}
\hline & $\begin{array}{c}\text { Razón de } \\
\text { posibilidades bruta }\end{array}$ & $\begin{array}{r}\text { Razón } \\
\text { correc }\end{array}$ & $\begin{array}{l}\text { le posibilidades } \\
\text { ida (IC95\%)a }\end{array}$ \\
\hline \multicolumn{4}{|l|}{ Gingivitis } \\
\hline Parto pretérmino & 1,00 & 0,98 & $(0,68-1,41)$ \\
\hline Peso bajo al nacer & 0,99 & 0,96 & $(0,68-1,37)$ \\
\hline Preeclampsia & 1,02 & 0,99 & $(0,69-1,43)$ \\
\hline \multicolumn{4}{|c|}{ Enfermedad periodontal grave } \\
\hline Parto pretérmino & 1,41 & 1,40 & $(0,90-2,17)$ \\
\hline Peso bajo al nacer & 1,53 & 1,51 & $(0,99-2,30)$ \\
\hline Preeclampsia & 1,05 & 1,09 & $(0,67-1,75)$ \\
\hline
\end{tabular}

${ }^{a}$ Intervalo de confianza de $95 \%$.

parto pretérmino $(\mathrm{RP}=0,98$; IC95\%: 0,68 a 1,41), el bajo peso al nacer ( $R P=$ 0,96; IC95\%: 0,68 a 1,37) la preeclampsia $(R P=0,99$; IC95\%: 0,69 a 1,43) (cuadro 3).

En total, 457 mujeres eran fumadoras; sin embargo, no se observó asociación alguna entre ser fumadora y el parto pretérmino $(R P=1,16$; IC95\%: 0,62 a 2,17), el bajo peso al nacer ( $\mathrm{RP}=$ 1,48; IC95\%: 0,82 a 2,68) o la preeclampsia $(R P=0,92$; IC95\%: 0,50 a 1,71). $\mathrm{Al}$ restringir este análisis a las $193 \mathrm{mu}-$ jeres que fumaban más de 10 cigarrillos diarios tampoco se encontró una asociación estadísticamente signifi- cativa con el parto pretérmino $(\mathrm{RP}=$ 1,15; IC95\%: 0,46 a 2,93), el bajo peso al nacer $(\mathrm{RP}=1,78$; IC95\%: 0,77 a 4,17) o la preeclampsia ( $\mathrm{RP}=0,87$; IC95\%: 0,36 a 2,12). El riesgo de dar a luz un niño con bajo peso al nacer en el subgrupo de fumadoras de más de 10 cigarrillos diarios fue significativamente mayor en el grupo de mujeres que tenían EP grave en comparación con las que tenían una salud bucal buena $(\mathrm{RP}=3,81$; IC95\%: 1,46 a 10,05; $P=0,001$ ).

Se confirmó que la anemia (presente en 556 de las mujeres participantes) es un factor de riesgo de bajo peso al nacer $(R P=1,74$; IC95\%: 1,03 a 2,94; 
CUADRO 4. Asociación entre la enfermedad periodontal y el desenlace del parto, controlado por la anemia ${ }^{a}$

\begin{tabular}{lccc}
\hline & $\begin{array}{c}\text { Razón de } \\
\text { posibilidades bruta }\end{array}$ & $\begin{array}{c}\text { Razón de posibilidades } \\
\text { corregida }(\text { IC95\%) }\end{array}$ \\
\hline $\begin{array}{l}\text { Gingivitis } \\
\quad \text { Parto pretérmino }\end{array}$ & 1,04 & 1,07 & $(0,74-1,55)$ \\
Peso bajo al nacer & 1,09 & 1,12 & $(0,78-1,60)$ \\
$\quad$ Preeclampsia & 1,02 & 1,00 & $(0,60-1,64)$ \\
Enfermedad periodontal grave & 1,33 & 1,39 & $(0,85-2,25)$ \\
$\quad$ Parto pretérmino & 1,59 & 1,64 & $(1,07-2,52)$ \\
Peso bajo al nacer & 0,96 & 0,95 & $(0,66-1,35)$ \\
Preeclampsia & & &
\end{tabular}

a Se contó con la información sobre la anemia en 1482 mujeres.

${ }^{\mathrm{b}}$ Intervalo de confianza de $95 \%$.

CUADRO 5. Efecto de la anemia sobre la asociación entre la enfermedad periodontal y el desenlace del parto

\begin{tabular}{|c|c|c|c|c|}
\hline & \multicolumn{2}{|c|}{ Mujeres con anemia } & \multicolumn{2}{|c|}{ Mujeres sin anemia } \\
\hline & $\mathrm{RP}^{\mathrm{a}}$ & IC95\% ${ }^{b}$ & $\mathrm{RP}$ & IC95\% \\
\hline \multicolumn{5}{|l|}{ Gingivitis } \\
\hline Parto pretérmino & 1,42 & $(0,83-2,42)$ & 0,82 & $(0,49-1,38)$ \\
\hline Peso bajo al nacer & 1,74 & $(1,03-2,94)$ & 0,73 & $(0,44-1,21)$ \\
\hline Preeclampsia & 0,99 & $(0,52-1,88)$ & 0,93 & $(0,60-1,44)$ \\
\hline \multicolumn{5}{|c|}{ Enfermedad periodontal grave } \\
\hline Parto pretérmino & 2,33 & $(1,29-4,58)$ & 0,80 & $(0,37-1,69)$ \\
\hline Peso bajo al nacer & 3,11 & $(1,62-5,97$ & 0,78 & $(0,38-1,60)$ \\
\hline Preeclampsia & 0,97 & $(0,37-2,48)$ & 1,01 & $(0,55-1,82)$ \\
\hline
\end{tabular}

${ }^{a}$ Razón de posibilidades.

b Intervalo de confianza de $95 \%$.

$P=0,02$ ). Al ajustar según la presencia de anemia en la madre, no se modificó la asociación entre los distintos desenlaces del parto y la EP (cuadro 4). A su vez, la anemia se comportó como una fuerte variable modificadora, ya que en las mujeres con anemia se fortaleció la asociación entre la EP por un lado, y el parto pretérmino y el bajo peso al nacer por el otro (cuadro 5).

\section{DISCUSIÓN}

A partir de 1996, diversos autores han estudiado la relación entre la enfermedad periodontal y algunas enfermedades sistémicas. El grupo pionero en este tipo de estudio fue el de la Uni- versidad de Carolina del Norte, Estados Unidos de América, encabezado por Offenbacher $(16,25)$, quien describió el papel desempeñado por los mediadores sistémicos de la infección periodontal y postuló que las embarazadas con EP tenían 7,5 a 7,9 veces mayor riesgo de dar a luz niños con bajo peso que las mujeres sin esa enfermedad. Otros estudios de casos y controles encontraron un aumento de 2 a 7 veces en el riesgo de parto pretérmino o de recién nacido de bajo peso $(16,25-32)$. Algunos estudios de cohortes también observaron un aumento de este riesgo entre 3 y 7 veces (33-37). En cuanto a la preeclampsia, se ha descrito que el riesgo se duplica en las mujeres con EP grave o progresión de la EP durante el embarazo (38). Algunos estudios de revisión confirman estos postulados (39-43).

El presente estudio, basado en el análisis de 1562 mujeres, solo mostró una tendencia a aumentar del riesgo de tener un parto pretérmino o hijos con peso bajo al nacer en las mujeres con EP grave, pero esta no fue estadísticamente significativa, excepto cuando además la mujer fumaba más de 10 cigarrillos diarios. No se encontró que las mujeres con EP tuvieran un riesgo significativamente mayor de preeclampsia.

Aunque la presencia de anemia fortaleció la asociación entre la EP y el riesgo de parto pretérmino y de dar a luz hijos con peso bajo al nacer, no influyó en los resultados del estudio. Cuando se ajustó según la condición de fumadora, no se encontraron cambios significativos en los resultados.

La fuerza de este estudio radica en el número de mujeres estudiadas, la prolija protocolización y el cuidadoso cumplimiento de sus pautas. Su mayor debilidad deriva de su diseño transversal, debido a la limitada capacidad de este tipo de estudios para detectar posibles asociaciones entre la exposición (EP) y los desenlaces. Se estima que la exclusión del análisis de 9\% de las mujeres por falta del diagnóstico odontológico no afectó a los resultados, ya que las causas de esa exclusión no dependieron de las variables estudiadas.

Otros estudios recientes realizados en países en desarrollo tampoco han confirmado la relación entre la EP y el nacimiento de niños con bajo peso (44). En cuanto a la asociación entre la EP y los nacimientos pretérmino, no se puede descartar la influencia de variables confusoras (45).

En el presente estudio no se encontró una asociación significativa entre la EP y el parto pretérmino, el bajo peso al nacer o la preeclampsia. Para avanzar en el estudio de la relación entre la EP y los sucesos perinatales imputados, es necesario determinar fehacientemente y en diversos ambientes (un estudio multicéntrico) si tal asociación existe y, de así confirmarse, investigar si con la prevención y el tratamiento de la EP durante el embarazo se reduce la incidencia de tales sucesos. 
Agradecimientos. A Lionel Schulman, Milton Klun, Yamil Partida y Silvina Perelló por su colaboración en el trabajo de obstetricia. A Marta Pasqua- rella, Antonio Walter, Cristian Sclavi, Claudio Sánchez, Yamil Fulgenzi, David Rodríguez, Juan Palmucci, Gonzalo Fernández, Marcela Arnaudo, Ma- tías Anghileri y Gladys Marcos por su colaboración en el trabajo de odontología. A Roberto Luis Giménez por su asesoramiento, estímulo y conocimientos.

\section{REFERENCIAS}

1. Newman M. Genetic, environmental, and behavioral influences on periodontal infections. Compend Contin Educ Dent. 1998;19:25-31.

2. Garrido del Cabo N, Carrión B, Ramos Barbosa I. Periodoncia para el odontólogo de práctica general. Enfermedad periodontal y embarazo. Periodoncia. 1999;9:31-40.

3. Amar S, Chung KM. Influence of hormonal variation on the periodontium in women. Periodontol 2000. 1994;6:79-87.

4. Jansen J, Liljemark W, Bloomquist C. The effect of female sex hormones on subgingival plaque. J Periodontol. 1981;52:599-602.

5. Kornman K, Loesche WJ. The subgingival microflora during pregnancy. J Periodontal Res. 1980;15:111-22.

6. Kornman K, Loesche J. Effects of estradiol and progesterone with Bacteroides melaninogenicus and Bacteriodes Gingivalis. Infect Inmun. 1982; 35(1):256-63.

7. Jonsson R, Howland BE, Bowden GHW. Relationship between periodontal health, salivary steroids, and Bacteroides intermedius in males, pregnant and non-pregnant women. J Dent Res. 1988;67:1062-8.

8. El-Attar TMA, Lin S, Tira DE. The relationship between the concentration of female sex steroids and prostaglandin production by human gingiva in vitro. Prostaglandins Leukot Med. 1982;8:447-58.

9. Hugoson A. Gingival inflammation and female sex hormones. J Periodontol. 1970;5:1-18.

10. Mohamed AH, Waterhouse JP, Friederici HHR. The microvasculature of the rabbit gingiva is affected by progesterone. An ultrastructural study. J Periodontol. 1974;45:50-60.

11. Loe H. Periodontal changes in pregnancy. J Periodontol. 1965;36:209-17.

12. Damare SM, Wells S, Offenbacher S. Eicosanoids in periodontal diseases: potential for systemic involvement. Adv Exp Med Biol. 1997:433:23-35.

13. Mustafa M, Wondimu B, Bakhiet M, Modeer T. Production of Rantes/CCL5 in human gingival fibroblasts challenged with tumour necrosis factor alpha. Eur J Oral Sci. 2001;109: 44-9.

14. Garrido de Cabo N, Blanco Carrión J, Ramos Barbosa I. Periodoncia para el odontólogo de práctica general. Periodoncia. 1999;9:31-40.

15. Dörtbudak O, Eberhardt MU, Persson UM. Periodontitis, a marker of risk pregnancy for preterm birth. J Clin Periodontol. 2005;32: $45-52$.

16. Offenbacher S, Katz V, Fertik G, Collins J, Boyd D, Maynor G, et al. Periodontal infection as a possible risk factor for preterm low birth weight. J Periodontol. 1996;67:103-13.

17. Champagne $\mathrm{CM}$, Madianos PN, Lieff $S$, Murtha AP, Beck JD, Offenbacher S. Periodontal medicine: emerging concepts in preg2003;91:215-20. nancy outcomes. J Int Acad Periodontol. 2000; 2:9-13.

18. Costa O, De la Cruz G, Sarudiansky A. Enfermedad coronaria diagnosticada por cinecoronariografía y su relación con la enfermedad periodontal. Rev Asoc Odontol Argentina.

19. Paquette DW. The periodontal infectionsystemic disease link: a review of the truth or myth. J Int Acad Periodontol. 2002;4:101-9.

20. Sacnnapieco FA. Position paper of American Academy of Periodontology: periodontal disease as a potential risk factor for systemic disease. J Periodontol. 1998;69:841-50.

21. Pihlstrom BL, Michalowicz BS, Johnson NW. Periodontal diseases. Lancet. 2005;366: 1809-20.

22. Williams CE, Davenport ES, Sterne JA, Sivapathasundaram V, Fearne JM, Curtis MA. Mechanisms of risk in preterm lowbirthweight infants. Periodontol. 2000;23: 142-50.

23. Cohen W, Rose L. The periodontal-medical risk relationship. Compend Contin Educ Dent. 1998;19:9-24.

24. Capurro H, Konichezky S, Fonseca D, Caldeyro-Barcia R. A simplified method for diagnosis of gestational age in the newborn infant. J Pediatr. 1978;93(1):120-2.

25. Offenbacher S. Periodontal diseases. Pathogenesis. Ann Periodontol. 1996;1:821-78.

26. Davenport ES, Williams CE, Sterne JA, Sivapathasundram V, Fearne JM, Curtis MA. The East London Study of Maternal Chronic Periodontal Disease and Preterm Low Birth Weight Infants: study design and prevalence data. Ann Periodontol. 1998;3:213-21.

27. Davenport ES, Williams CE, Sterne JA, Murad S, Sivapathasundram V, Curtis MA. Maternal periodontal disease and preterm low birth weight: case-control study. J Dent Res. 2002; 81:313-8.

28. Jeffcoat MK, Geurs NC, Reddy MS, Cliver SP Goldenerg RL, Hauth JC. Periodontal infection and preterm birth: results of a prospective study. J Am Dent Assoc. 2001;132:875-80.

29. Offenbacher S, Jared HL, O'Reilly PG, Wells SR, Salvi GE, Lawrence HP, et al. Potential pathogenic mechanisms of periodontitis associated pregnancy complications. Ann Periodontol. 1998;3:233-50.

30. Romero BC, Chiquito CS, Elejalde LE, Bernardoni CB. Relationship between periodontal disease in pregnant women and the nutritional condition of their newborns. J Periodontol. 2002;73:1177-83.

31. Offenbacher S, Beck JD. Periodontitis: a potential risk factor for spontaneous preterm birth. Comp Contin Educ Dent. 1998;19:32-9.

32. Moore S, Randhawa MS, Ide M. A casecontrol study to investigate an association be- tween adverse pregnancy outcome and periodontal disease. J Clin Periodontol. 2005;32: 1-5.

33. López NJ, Smith PC, Gutiérrez J. Higher risk of preterm birth and low birth weight in women with periodontal disease. J Dent Res. 2002:81:58-63.

34. Mitchell-Lewis D, Engebretson SP, Chen J, Lamster IB, Papapanou PN. Periodontal infections and pre-term birth: early findings from a cohort of young minority women in New York. Eur J Oral Sci. 2001;109:34-9.

35. Offenbacher $S$, Lieff $S$, Bogges KA, Murtha AP, Medianos PM, Champagne CM, et al. Maternal periodontitis and prematurity. Part I: Obstetric outcome of prematurity and growth restriction. Ann Periodontol. 2001;6:164-74.

36. López NJ, Smith PC, Gutiérrez J. Periodontal therapy may reduce the risk of preterm low birth weight in women with periodontal disease: a randomized controlled trial. J Periodontol. 2002;73:911-24.

37. Dasanayake AP, Russell S, Boyd D, Madianos PN, Forster Tornquist, et al. Preterm low birth weight and periodontal disease among African Americans. Dent Clin North Am. 2003;47: $115-25$.

38. Boggess KA, Lieff S, Murtha A, Moss K, Beck J, Offenbacher S. Maternal periodontal disease in associated with an increased risk for preeclampsia. Obstet Gynecol. 2003;101: 227-31.

39. Garrido de Cabo N, Blanco Carrión J, Ramos Barbosa I. Periodoncia para el odontólogo de práctica general. Periodoncia. 1999;9:31-40.

40. Garbero I, Cárdenas B, Ida I. Papel de la enfermedad periodontal en el parto pretérmino de bajo peso. Periodoncia. 2001;11:51-8.

41. Jeffcoat MK, Geurs NC, Reddy MS, Goldenberg RL, Hauth JC. Current evidence regarding periodontal disease as a risk factor in preterm birth. Ann Periodontol. 2001;6:183-8.

42. Offenbacher S, Beck J. Periodontitis: a potential risk factor for spontaneous preterm birth. Comp Contin Educ Dent. 1998;19:32-9.

43. McGaw T. Periodontal disease and preterm delivery of low-birth-weight infants. J Can Dent Assoc. 2002;68:165-9.

44. Lunandelli AN, Peres MA. Is there an association between periodontal disease, prematurity and low birth weight? A population-based study. J Clin Periodontol. 2005;32: 938-46.

45. Khader YS, Ta'ani Q. Periodontal diseases and the risk of preterm birth and low birth weight: a meta-analysis. J Periodontol. 2005; 76:161-5

Manuscrito recibido el 6 de agosto de 2004. Aceptado para publicación, tras revisión, el 6 de marzo de 2006. 
ABSTRACT Objectives. To determine if periodontal disease (PD) is a risk factor for premature labor, low birth weight, or preeclampsia, and to look for a potential association between the different clinical forms of PD and the obstetric results indicated.

Periodontal disease: Is it a risk factor for premature labor, low birth weight or preeclampsia?

Key words Periodontal diseases; labor, premature; birth weight; preeclampsia; Argentina.
Methods. This is an analytical, cross-sectional and prospective study of all women who gave birth in Dr. José Penna Hospital, in the city of Bahía Blanca, Argentina, between 1 February and 18 July 2003 and between 1 March and 31 May 2004. Women who met the inclusion criteria (being over 18 years old, having at least 18 teeth, not being diabetic, and having had children who survived labor) underwent a dental exam to look for hemorrhage when teeth were probed, loose teeth, or inflammation of the gums, and any loss of insertion was measured clinically. Odds ratios (OR) and 95\% confidence intervals (95\% CI) were calculated, and the results were adjusted according to smoking status and the presence or absence of anemia.

Results. A total of 2003 births and 1982 labor episodes were registered. In all, 420 women $(21.2 \%)$ were excluded from the study because they did not meet the inclusion criteria or because no diagnostic exam could be carried out. Of the 1562 women who were examined, $809(51.8 \%)$ had at least one of the problems being sought; of these women, $274(17.5 \%)$ had severe periodontal disease, and $535(34.3 \%)$ had gingivitis. In all, there were $149(9.5 \%)$ cases of premature labor; 161 (10.3\%) cases of low birth weight, and $157(10.0 \%)$ cases of preeclampsia. No association was noted between PD and premature labor (OR $=1.06$; $95 \% \mathrm{CI}$ : 0.74 to 1.50$)$, low birth weight $(\mathrm{OR}=1.05 ; 95 \% \mathrm{CI}: 0.74$ to 1.47$)$, or preeclampsia (OR $=0.99 ; 95 \% \mathrm{CI}: 0.70$ to 1.40$)$. The risk of giving birth to an infant with low birth weight in the subgroup of women who smoked more than 10 cigarettes a day was greater among women who had severe PD than among those who had good oral health $(\mathrm{OR}=3.81 ; 95 \% \mathrm{CI}: 1.46$ to $10.05 ; P=$ $0.001)$. Anemia was found to be a risk factor for low birth weight $(\mathrm{OR}=1.74$; IC 95\%: 1.03 to $2.94 ; P=0.02$ ).

Conclusions. No significant association was found between PD and premature labor, low birth weight, or preeclampsia.
Clasificación Internacional de Enfermedades Aplicada a Odontología y Estomatología Tercera edición (3)

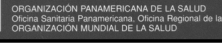

1996, $238 \mathrm{pp}$. ISBN 9275315620 Código: PC 562 Precio: US\$ 22.00 en América Latina y el Caribe y US\$ 32.00 en el resto del mundo

\section{Clasificación Internacional de Enfermedades Aplicada a Odontología y Estomatología (CIE-OE), 3. ${ }^{a}$ ed.}

Esta tercera edición de la Clasificación internacional de enfermedades aplicada a odontología y estomatología (CIE-OE) tiene por objeto proveer bases prácticas y útiles para codificar y clasificar datos relativos a los trastornos bucales y dentarios. La CIE-OE se deriva directamente de la Décima revisión de la Clasificación internacional de enfermedades (CIE-10), y ha sido concebida como parte de la "familia" de clasificaciones de enfermedades y problemas relacionados con la salud.

En la CIE-OE se incluyen todas las enfermedades y trastornos que se presentan en la cavidad bucal y estructuras adyacentes o que de algún modo las afectan. La mayoría de las clasificaciones provistas por la CIE-10 han sido subdivididas y ampliadas para incluir un quinto carácter con el fin de llamar la atención de los profesionales de la salud bucal sobre la necesidad de realizar un diagnóstico detallado para cada paciente y contar con un registro estandarizado para todos los diagnósticos que facilite la comparación de los datos a nivel internacional. Se espera que la CIE-OE contribuya de forma importante a la recolección de datos epidemiológicos sobre las enfemedades bucales menos frecuentes, para las cuales no hay buenos métodos de vigilancia.

http://publications.paho.org・Fax: (301) 206-9789・Correo electrónico: paho@pmds.com 